\title{
Pesquisa e Extensão em Saúde e a Aprendizagem nos Níveis Cognitivo e Afetivo
}

\section{Research and Extension in Health and Cognitive and Affective Learning Levels}

João Luiz Gurgel Calvet da Silveira Karla Ferreira Rodrigues ${ }^{I}$ Mônica Shishido ${ }^{I}$ Patrícia Moraes ${ }^{I}$

\section{PALAVRAS-CHAVE \\ - Aprendizagem; \\ - Educação Superior; \\ - Integralidade em Saúde; \\ - Recursos Humanos em Saúde; \\ - Educação Médica.}

\section{RESUMO}

O objetivo deste estudo foi comparar a aprendizagem nos níveis cognitivo e afetivo entre alunos bolsistas do programa PET-Saúde e alunos que não tiveram experiência em atividades de pesquisa e extensão. O método utilizado foi um estudo exploratório comparativo com 14 estudantes dos cursos de Medicina, Medicina Veterinária, Odontologia, Nutrição, Fisioterapia, Enfermagem e Educação Física da Universidade Regional de Blumenau. Os temas abordados foram integralidade do cuidado em saúde, pesquisa e extensão. Utilizou-se abordagem qualitativa por meio da técnica de entrevista semiestruturada. A análise foi realizada por meio da taxonomia de Bloom.Os Alunos envolvidos com as atividades de pesquisa e extensão no programa PET-Saúde atingiram maiores níveis da taxonomia de Bloom nos domínios cognitivo e afetivo em comparação com os outros estudantes. A experiência dos estudantes nos grupos PET-Saúde parece contribuir para uma aprendizagem diferenciada nos cursos da saúde.

\section{ABSTRACT}

The objective of this study was to compare the cognitive and affective learning levels between scholarship students of the PET-Saúde program and other students who have had no experience in research and extension activities. The method used was a comparative study involving 14 students of Medicine, Veterinary Medicine, Dentistry, Nutrition, Physiotherapy, Nursing and Physical Education from the Regional University of Blumenau. The topics covered were comprehensive health care, research and extension. A qualitative approach was used involving semi-structured interviews. The analysis was performed using Bloom's Taxonomy. The students involved with research and extension activities in the PET-Saúde program achieved higher levels of Bloom's Taxonomy considering the Cognitive and Affective Domains, compared to the other students. The experience of students in PET-Saúde seems to contribute toward a differentiated learning in health courses. 


\section{A FORMAÇÃO NA SAÚDE}

A formação dos profissionais de saúde é reconhecida por organismos internacionais como um importante fator para a saúde da população por seu impacto direto nos sistemas de saúde, seja na atenção ou na gestão dos serviços, assim como na definição de políticas públicas. Por este motivo, várias iniciativas buscam aproximar a formação em saúde, representada pelas universidades, e o serviço, representado pelas instâncias executivas do Sistema Único de Saúde (SUS), principalmente as secretarias municipais de Saúde. Nesse contexto, importa conhecer, a partir dos sujeitos do aprendizado, como as diversas dimensões integrantes do cotidiano da universidade, potencializadas na proposta dos grupos PET-Saúde, contribuem para a formação desses futuros profissionais e em que nível este aprendizado se expressa para estes sujeitos.

No início do século XIX, o médico norte-americano Abraham Flexner publicou o Relatório Flexner, cujo trabalho influenciou de forma determinante o ensino das profissões da área da saúde, principalmente nos EUA e Brasil. Esse relatório determinou grande ênfase em disciplinas ou especialidades ambientados, em sua maior parte, dentro de hospitais, gerando um currículo segmentado em ciclos básico e profissional ${ }^{1}$. Nos anos 1940, o modelo flexneriano chegou ao Brasil e influenciou os cursos de Medicina, Enfermagem e Odontologia, além de outros já existentes².

A partir das críticas ao modelo flexneriano, surgem propostas inovadoras, que buscam superar o modelo hospitalocêntrico melhorando a relação da universidade com a sociedade e suas demandas, com destaque do movimento de Integração-Docente-Assistencial (IDA) e dos pressupostos da Medicina Comunitária ${ }^{3}$.

Nessa mesma época, a formação dos profissionais de saúde foi alvo de críticas que buscavam valorizar a interdisciplinaridade do conhecimento ${ }^{4}$. Nesse sentido, a profissionalização, gestada e gerida no ambiente acadêmico, deveria se aproximar da realidade social e considerar as demandas dos serviços, constituindo vínculos humanísticos e consistentes com a população ${ }^{5}$. A inserção ensino-serviço ocorrida nesse período não foi suficiente para modificar a prática fragmentada em especialidades e o ensino hospitalocêntrico. Assim, em 1978, a Reunião Alma-Ata instigou a discussão sobre a Atenção Primária em Saúde, influenciando o movimento da Reforma Sanitária no Brasil ${ }^{2}$

Grandes mudanças ocorreram no Brasil nas décadas de 1980 e 1990 devido à Constituição de 1988 e ao advento do Sistema Único de Saúde (SUS) ${ }^{6}$. Ademais, em 1991, a Comissão Interinstitucional Nacional de Avaliação das Escolas Médicas (Cinaem) foi criada pela Associação Brasileira de Ensino
Médico (Abem), Conselho Federal de Medicina e outras nove instituiç̃ões ${ }^{7}$ com o intuito de avaliar o ensino nas escolas médicas. Ainda nos anos 1990, a Cinaem obteve alguns resultados que favoreceram a criação do Programa UNI (Uma Nova Iniciativa na formação dos profissionais de saúde), cujo objetivo era desenvolver estratégias para intensificar as mudanças na formação dos profissionais de saúde incluindo a comunidade. Desse modo, pode-se afirmar que os projetos UNI e IDA (Integração Docente-Assistencial) apresentavam ideais em comum e algum êxito por certo tempo ${ }^{2}$. Em 1997, surge a Rede Unida, que amplia essas demandas para a formação das demais profissões da saúde, incorporando os atores e princípios dos projetos UNI e IDA, que almejavam potencializar as capacidades de transformação, principalmente as coletivas, a partir do saber e fazer compartilhados ${ }^{8}$. Além de discutir as Diretrizes Curriculares, a Rede Unida participou do Programa de Incentivo a Mudanças Curriculares nos Cursos de Medicina (Promed), que foi base para o surgimento do Programa Nacional de Reorientação da Formação Profissional em Saúde (Pró-Saúde) em 3 de novembro de $2005^{9}$.

\section{A ESTRATÉGIA DE EDUCAÇÃO PELO TRABALHO: O PET DA SAÚDE}

Como estratégia complementar, integrada ao Pró-Saúde ${ }^{10}$, foi criado o Programa de Educação pelo Trabalho para a Saúde (PET-Saúde), instituído pela portaria interministerial noำ 1.802, de 26 de agosto de 2008, no qual se firma uma articulação entre os ministérios da Saúde e da Educação a fim de formar profissionais com perfil apropriado à Atenção Básica no contexto do SUS ${ }^{11}$.

Embasado no princípio da educação tutorial, o PET-Saúde constitui uma alternativa estratégica à formação academicista ou exclusivamente profissional desvinculada da realidade, integrando a universidade, o serviço e a comunidade num processo em que professores tutores orientam estudantes por meio de metodologias científicas com responsabilidade ética e social, repensando as práticas pedagógicas ${ }^{11}$. Entre os principais objetivos do PET-Saúde destaca-se a integração ensino-serviço à procura de respostas para as demandas reais da população brasileira a partir da formação adequada de profissionais da saúde, para atender às necessidades do SUS e da comunidade, qualificando, simultaneamente a prestação de serviços e a produção de conhecimento para fortalecer o SUS ${ }^{11,12}$.

Nesse sentido, relatos na literatura apontam na proposta do PET-Saúde a possibilidade de uma vida estudantil mais rica em experiências e com maior aproveitamento do ensino e da capacidade de aprendizagem, além da melhoria da qualidade do curso por meio de sua inserção nas atividades aca- 
dêmicas. Essa proposta pode ainda contribuir para o processo ensino-aprendizagem, pois intensifica a integração entre acadêmicos e docentes ${ }^{13}$.

No que se refere à prática de pesquisa nos cursos de graduação, antes de constituir uma exigência meramente acadêmica, tal atividade pode representar uma possibilidade de formação diferenciada, constituindo um princípio educativo para uma formação mais crítica e contextualizada, baseada nos princípios do método científico e na investigação do conhecimento renovado pela pesquisa ${ }^{14}$.

Assim, em consonância com o PET-Saúde, as Diretrizes Curriculares Nacionais (DCN) focalizam a construção de um profissional da saúde (Medicina, Odontologia, Medicina Veterinária, Psicologia, Educação Física, Enfermagem, Nutrição e Farmácia) por meio da articulação e interação entre ensino, pesquisa e extensão. A pesquisa visa construir um profissional que obtenha capacidades por meio do próprio ato de aplicar e participar das pesquisas e, além disso, saiba empregar os conhecimentos obtidos na prática profissional e compartilhe esses conhecimentos. A integração entre pesquisa e extensão se dá à medida que o acadêmico passa a aplicar na prática conceitos obtidos de forma teórica. Segundo as diretrizes, é preciso ainda uma implementação precoce das atividades práticas, nas quais os alunos possam utilizar diferentes cenários de ensino-aprendizagem ${ }^{15}$. Graças a essa variação de ambientes, é possível vivenciar uma gama de experiências e práticas de serviço diferenciadas, que propiciam um trabalho aprimorado e interdisciplinar.

O presente trabalho foi realizado a partir do projeto PET-Saúde de Blumenau (SC), fruto de uma parceria entre a Universidade Regional de Blumenau (Furb) e a Secretaria Municipal de Saúde de Blumenau (Semus), encontrando-se em sua terceira edição. Este estudo objetiva comparar o perfil de aprendizagem nos domínio cognitivo e afetivo de alunos participantes do PET-Saúde com o perfil de alunos de curso e fase correspondentes que nunca participaram de experiências de pesquisa ou extensão.

\section{PERCURSO METODOLÓGICO}

Este é um estudo exploratório comparativo, tendo como sujeitos sete pares de alunos pertencentes aos cursos de graduação do Centro de Ciências da Saúde: Medicina, Medicina Veterinária, Odontologia, Nutrição, Fisioterapia, Enfermagem e Educação Física. Os alunos participantes foram comparados em pares, e cada par de estudantes deveria obrigatoriamente estar cursando a mesma fase do mesmo curso, sendo um deles bolsista do projeto PET-Saúde, denominado "petiano", e outro sem nenhuma experiência prévia em atividades de pesquisa e extensão, denominado "não petiano".
As temáticas utilizadas para a abordagem dos alunos, por meio da técnica de entrevista semiestruturada, foram: "integralidade do cuidado em saúde", "pesquisa" e "extensão" no contexto da formação universitária, buscando registrar a definição conceitual e o significado atribuídos pelos sujeitos aos temas trabalhados.

Para aferir o nível cognitivo e afetivo emitido pelos entrevistados sobre os temas geradores, foi utilizada a taxonomia de Bloom ${ }^{16}$. Neste caso, os níveis de classificação são crescentes, ou seja, do mais simples para o mais complexo, nos dois domínios avaliados neste estudo ${ }^{16}$.

Esta metodologia de análise se baseia no princípio de que os processos caracterizados pela taxonomia devem representar resultados de aprendizagem, ou seja, cada categoria taxonômica representa o que o indivíduo aprende no campo conceitual, e não aquilo que ele já sabe, assimilado do seu contexto familiar ou cultural. Nesse contexto, o domínio cognitivo é o mais frequentemente utilizado em pesquisas com o objetivo de analisar os níveis de aprendizagem de discentes de acordo com a taxonomia dos objetivos educacionais de Bloom ${ }^{16,17}$.

Pressupõe-se que estes processos taxonômicos sejam integrados, isto é, sigam uma ordem cumulativa, em que uma categoria cognitiva depende da categoria anterior e, por sua vez, serve de base para a seguinte. Essas categorias são organizadas num gradiente em termos de complexidade dos processos mentais ${ }^{16}$ (Figura 1).

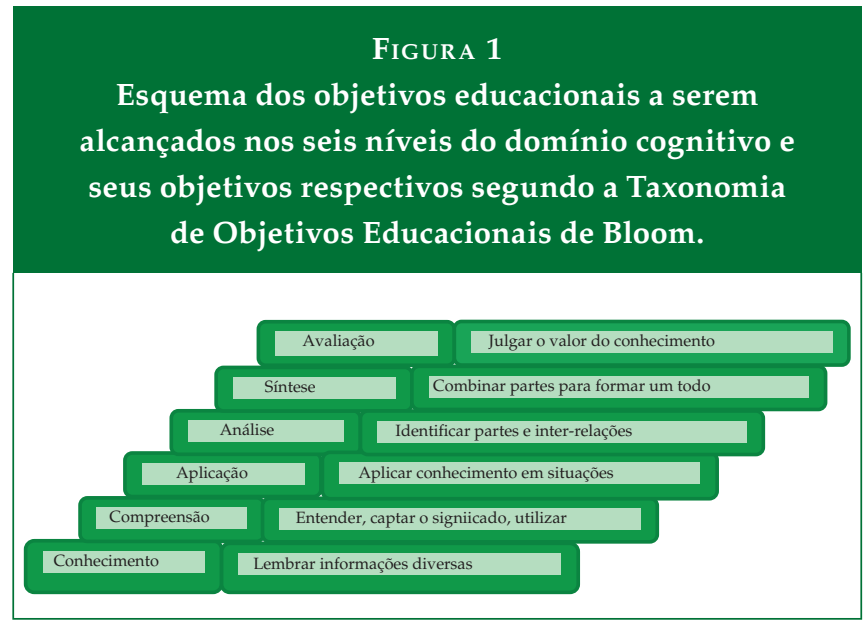

No tocante à parte afetiva, a avaliação busca relacionar sentimentos e posturas dos discentes que envolvam categorias ligadas ao desenvolvimento da área emocional e afetiva, que incluem comportamento, atitude, responsabilidade, respeito, emoção e valores. Da mesma forma, para ascender a uma nova categoria, é preciso ter obtido desempenho adequado na anterior, pois cada uma utiliza capacidades adquiridas nos ní- 
veis anteriores para serem aprimoradas. As categorias deste domínio são: recepção, resposta, avaliação, organização e internalização ${ }^{16}$ (Figura 2).
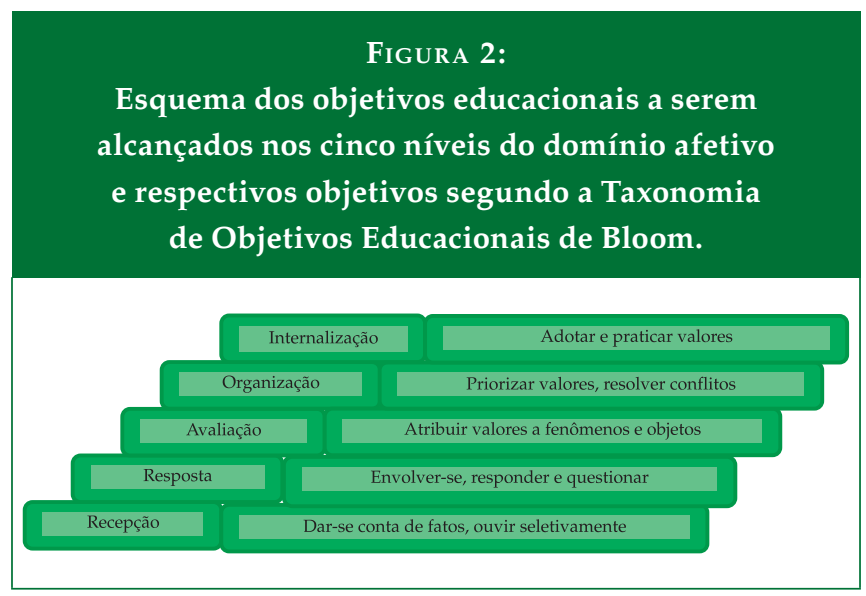

Para a classificação do domínio cognitivo, cada tema foi definido com base nas seguintes referências:

— Tema 1: Integralidade do cuidado: "cuidado de pessoas, grupos e coletividade percebendo o usuário como sujeito histórico, social e político, articulado ao seu contexto familiar, ao meio ambiente e à sociedade na qual se insere ${ }^{\prime 18}$ (p.336);

— Tema 2: Pesquisa: "Questionamento sistemático crítico e criativo, mais a intervenção competente na realidade, ou o diálogo crítico permanente com a realidade em sentido teórico e prático"19(p.34);
— Tema 3: Extensão: "[...] processo educativo, cultural e científico que articula o ensino e a pesquisa de forma indissociável e viabiliza a relação transformadora entre universidade e sociedade. [...] troca de saberes sistematizado, acadêmico e popular, terá como consequências a produção do conhecimento resultante do confronto com a realidade brasileira e regional, a democratização do conhecimento acadêmico e a participação efetiva da comunidade na atuação da universidade. [...] é um trabalho interdisciplinar que favorece a visão integrada do social" ${ }^{\prime 20}$ (p.5).

Após a transcrição das entrevistas, as falas foram classificadas nos diferentes níveis estabelecidos por Bloom com base nos objetivos educacionais e capacidades a adquirir das Figuras 1 e 2 .

O projeto de pesquisa foi aprovado pelo Comitê de Ética em Pesquisa com Humanos sob o protocolo 195/10.

\section{APLICANDO A TAXONOMIA DE BLOOM ÀS FALAS DOS ESTUDANTES}

A Tabela 1 descreve a classificação dos níveis alcançados entre os alunos "petianos" e "não petianos" nos domínios cognitivo e afetivo. De forma geral, os alunos "petianos", envolvidos com atividades de pesquisa e extensão, atingiram os níveis mais elevados da taxonomia de Bloom comparativamente aos "não petianos", para os domínios cognitivo e afetivo, considerando os três temas trabalhados nas entrevistas.

Quanto ao domínio cognitivo, observamos que o quinto nível (síntese) foi atingido somente por alunos "petianos" para os conceitos de Integralidade e Extensão, conforme as falas:

\begin{tabular}{|c|c|c|c|c|c|c|c|c|}
\hline \multirow{3}{*}{\multicolumn{2}{|c|}{ T }} & \multicolumn{5}{|c|}{$\begin{array}{l}\text { TAвELA } 1 \\
\text { sujeitos nos diferentes eixos temáticos. }\end{array}$} & & \\
\hline & & Acadêmicos & \multicolumn{3}{|c|}{ Aluno "petiano" } & \multicolumn{3}{|c|}{ Aluno "não-petiano" } \\
\hline & & Nível Eixo & $\begin{array}{l}\text { Integra- } \\
\text { lidade }\end{array}$ & Pesquisa & Extensão & $\begin{array}{l}\text { Integra- } \\
\text { lidade }\end{array}$ & Pesquisa & Extensão \\
\hline $\begin{array}{l}X \\
O\end{array}$ & \multirow{6}{*}{$\begin{array}{l}\text { Domínio } \\
\text { Cognitivo }\end{array}$} & Conhecimento & 1 & 1 & 2 & 1 & 5 & 1 \\
\hline $\mathbf{N}$ & & Compreensão & 1 & 1 & 1 & 1 & 1 & 1 \\
\hline $\mathbf{O}$ & & Aplicação & 4 & 1 & 2 & 1 & 1 & 1 \\
\hline $\mathbf{M}$ & & Análise & & 3 & & & & \\
\hline \multirow{2}{*}{$\begin{array}{l}\text { I } \\
\text { A }\end{array}$} & & Síntese & 1 & & 2 & & & \\
\hline & & Avaliação & & & & & & \\
\hline $\begin{array}{l}\text { D } \\
\text { E }\end{array}$ & $\begin{array}{l}\text { Alunos que não atingiram o } \\
\text { nível mínimo (cognitivo) }\end{array}$ & & & 1 & & 4 & & 4 \\
\hline \multirow{6}{*}{$\begin{array}{l}\text { B } \\
\text { L } \\
\text { O } \\
\text { O } \\
\text { M }\end{array}$} & \multirow{5}{*}{ Domínio afetivo } & Recepção & 3 & 2 & 1 & 1 & 3 & \\
\hline & & Resposta & 1 & 1 & 1 & & 1 & 2 \\
\hline & & Avaliação & & 3 & 1 & & 1 & \\
\hline & & Organização & & & & & & \\
\hline & & Internalização & 2 & 1 & 4 & & & \\
\hline & $\begin{array}{l}\text { Alunos que não atingiram o } \\
\text { nível mínimo (afetivo) }\end{array}$ & & 1 & & & 6 & 2 & 5 \\
\hline
\end{tabular}


"É ver o paciente como um todo, não só a doença dele, ver onde ele mora, o que ele sabe em relação a sua doença, como pode estar prevenindo, é ver ele como um todo mesmo [...] integral, você não vai tratar só a hipertensão, você tem que tratar ele, tem que ver onde ele mora, quais são as dificuldades[...] educação em saúde, muito utilizada na integralidade, não olhar só a doença, mas olhar o paciente, a família, a moradia, tudo". (sujeito 1 )

"Extensão, eu acho que você usa a pesquisa e o ensino para fazer a extensão, e através da extensão universitária você sai da universidade e consegue fazer atividades que vão transformar tanto a sociedade como quem está fazendo a extensão". (sujeito 7)

Ainda entre os "petianos", para o eixo temático Pesquisa, foi atingido o quarto nível (análise), conforme as falas:

"[...] pesquisa eu acho que é você fazer uma revisão sobre o assunto, antes de tudo, inteirar-se, coletar dados e ver se é isso mesmo, se os teus dados vão bater com os outros dados que você achou". (sujeito 6)

"[...] pra mim, pesquisa é quando você formaliza através de um método científico e de um tema central que você tem interesse, você vai fazer questionamentos e através deles conseguir uma resposta através de um trabalho científico". (sujeito 7)

Entre os alunos "não petianos", percebe-se um alcance máximo no terceiro nível do domínio cognitivo (aplicação) para os três eixos temáticos. Destaca-se ainda que a grande maioria desses alunos se manteve no primeiro nível do domínio cognitivo (conhecimento) para o eixo temático Pesquisa, o qual se limita à capacidade de memorização, sendo ilustrado nas falas:

"Cuidar de toda a situação sem nenhuma forma de discriminação, porque todo mundo merece cuidado". (sujeito 13)

"Análise de dados com um objetivo final, basicamente isso!" (sujeito 5)

"Extensão é a realização de atividades[...] atividades do conhecimento acadêmico, que você proporciona e fornece pra comunidade em geral". (sujeito 14)

No que se refere ao domínio afetivo, entre os alunos "petianos" houve maior frequência dos que atingiram o mais alto nível (quinto nível - internalização), especialmente em relação ao conceito de Extensão. Seguem as falas dos estudantes:
"Na faculdade, a gente tem uma troca também de experiências. Tem pessoas que trabalham no posto de saúde, que trabaIham em hospitais, tem essa troca de informações com quem já está na área,essa integralidade. Isso é muito importante para mim". (sujeito 1 )

"[pesquisa] é algo que eu vou acrescentar pra minha vida, tanto pessoal, profissional, acho que é de extrema importância; querendo ou não, acho que no nosso dia a dia a gente acaba tendo um pouquinho da pesquisa na nossa grade curricular [devido ao PET-Saúde]". (sujeito 2)

"Eu acho que vai muito mais além da minha profissão, ou a nós como estudantes, porque faz com que possa abrir outros caminhos, tu pode estar trabalhando em uma unidade de saúde, ou auxiliar em um hospital, ou tu estar fazendo uma visita domiciliar, então entende além do que só ficar dentro da universidade, tu vai além disso, e também acho muito importante". (sujeito 4)

Em comparação com as falas acima, os alunos não envolvidos em pesquisa e extensão apresentaram níveis mais elementares do domínio afetivo, limitando-se à capacidade de recepção (primeiro nível) para o conceito de Integralidade, capacidade de resposta (segundo nível) para o conceito de Extensão, e somente um aluno atingiu o terceiro nível (avaliação) para o conceito de Pesquisa. Um estudo que utilizou metodologia ativa e inserção no serviço apresentou resultado semelhante, com valorização do aprendizado pelos estudantes $^{21}$. Seguem as falas ilustrativas dos "não petianos":

"A importância da integralidade do cuidado é você desenvolver uma convivência harmoniosa e zelosa com as outras pessoas [...] é quando eu vejo que uma pessoa está fazendo alguma coisa errada, alguma coisa que venha prejudicar a ela, é alertar esta pessoa, zelando por ela e pelas outras pessoas". (sujeito 14)

"[...] vamos supor, na área da saúde com pesquisas, elas vão, principalmente, beneficiar a sociedade, quando são pesquisas benéficas que têm novas descobertas. Então, talvez elas não saibam como isso funciona, mas principalmente vai trazer benefícios para elas". (sujeito 9)

"Eu acho [extensão] que seria tipo depois da pesquisa e ter meio que formado aquele conhecimento, seria aplicar ele [...] vai ver se na prática vai dar certo". (sujeito 10)

Chamam a atenção os níveis relativamente mais baixos demonstrados pelos alunos "não petianos", principalmente no 
domínio afetivo. Este fato pode ser explicado pela necessária precedência da elaboração do conceito no campo cognitivo para uma internalização que possibilite um avanço no campo afetivo.

\section{POTENCIALIDADES DA PROPOSTA PET PARA A FORMAÇÃO EM SAÚDE}

A proposta dos grupos PET-Saúde atende aos princípios da Educação Interprofissional em Saúde e segue a tendência internacional de formar profissionais da saúde que apresentem como características: atendimento das necessidades locais e dos trabalhadores da saúde; inovação curricular e pedagógica baseada na educação tutorial; alinhamento entre a educação e o cuidado no serviço, ou integração ensino-serviço ${ }^{22}$.

Estudos sobre a experiência dos grupos PET-Saúde apresentam como resultado uma formação crítica e ampliada a partir do processo saúde-doença e próxima dos princípios do SUS. Assim como no presente estudo, foram observadas as seguintes potencialidades dessa proposta: ressignificação do SUS; aumento da capacidade crítica dos alunos, potencialização da interdisciplinaridade e concretização da integração ensino-serviço-comunidade ${ }^{23-26}$. Os resultados desses estudos traduzem o potencial dos projetos PET-Saúde como um poderoso instrumento de indução de mudanças nas concepções dos profissionais de saúde.

Facchini et al..$^{27}$ defendem a importância da pesquisa na Atenção Básica em saúde. A prática de pesquisa, conforme desenvolvida nos grupos PET-Saúde, pode qualificar a formação e o processo de trabalho no SUS com base nos dados gerados. Apresenta potencial para ampliar a efetividade das ações da equipe de ESF, incorporando as inovações em ciência e tecnologia apropriadas à realidade, tendo a universidade - por intermédio dos estudantes e docentes em interação com os preceptores, trabalhadores do SUS, gestão e comunidade - um papel relevante nesse processo.

Um estudo baseado na prática de extensão na formação em saúde revela o potencial dessa prática em produzir o cuidado integral a partir da indissociabilidade entre ensino-pesquisa-extensão, com valorização da extensão como espaço de vivência "dialógica, multiprofissional e socialmente compromissada", em concordância com os relatos obtidos no presente estudo e integrando os princípios e conceitos abordados nesta pesquisa ${ }^{28}$.

A extensão, de certa forma, é negligenciada nas universidades, que buscam apenas ensinar técnicas e habilidades mecânicas ${ }^{29}$. Contrapondo-se a esta tendência na área da saúde, a extensão constitui uma dimensão preponderante na proposta dos grupos PET-Saúde, na qual os acadêmicos e profissionais buscam orientar suas práticas mediante contato direto com a população.

\section{CONSIDERAÇÕES FINAIS}

A experiência dos estudantes nos grupos PET-Saúde parece contribuir para uma aprendizagem diferenciada nos cursos da área da saúde, considerando os domínios cognitivo e afetivo. Ao trabalharem atividades de ensino, pesquisa e extensão de forma indissociada em grupos interdisciplinares em situações reais de trabalho, envolvendo equipes de saúde e comunidade, as práticas de ensino-aprendizagem apresentam potencial para atender às tendências de mudança na formação em saúde nos cenários nacional e internacional. O presente estudo propõe a perspectiva de maior aprofundamento sobre o tema do ensino na área da saúde, para um aprendizado mais crítico, significativo e capaz de favorecer o desenvolvimento da autonomia nos sujeitos da aprendizagem.

Projeto financiado pelo edital PET-Saúde nº 12/2008 da Secretaria de Gestão do Trabalho e da Educação na Saúde SGTES - Ministério da Saúde. Cadastro no Sipex-Furb no $467 / 2009$.

Projetoa provado pelo Comitê de Ética na Pesquisa em Seres Humanos da Furb pelo parecer n⿳0 043/2009.

\section{REFERÊNCIAS}

1. Sakai MH. Recursos Humanos em Saúde. In: Andrade SM, Soares DA, Cordoni LJR, org. Bases da Saúde Coletiva. Londrina: EdUEL; 2001. p.111-24.

2. Feuerwerker LCM, Marsiglia R. Estratégias para mudanças na formação de RHs com base nas experiências IDA/ UNI. Divulgação em saúde para debate1996; (12):24-8.

3. Santana JP, Campos FE, Sena RR. Formação profissional em saúde: desafios para a universidade. In: Santana JP, Castro JL, organizadores. Capacitação em desenvolvimento de recursos humanos de saúde. Natal: EDUFRN; 1999. p.109-23.

4. Garcia MAA, Pinto ATBCS, Odoni APC, Longhi BS, Machado LI, Linek MDS, Costa NA. Interdisciplinaridade e integralidade no ensino em saúde. Rev. Ciênc. Méd. 2006; Campinas,15(6):473-85.

5. Feuerwerker LCM, Sena RR. Contribuição ao movimento de mudança na formação profissional em saúde: uma avaliação das experiências UNI. Interface 2002; 6(10):37-50.

6. Merhy EE, Feuerwerker LCM, Ceccim RB. Educación Permanente en Salud: una estrategia para intervenir en la micropolítica del trabajo en salud. SaludColectiva 2006; 2(2):147-60

7. Santos NR, org. A prática do controle social através dos conselhos de saúde. Divulgação em saúde para debate 2000; (22):71-91.

8. Rangel ML, Vilasbôas AL. Rede UNIIDA: Breve histórico, concepção, organização e estratégias de ação. Divulgação em saúde para debate 1996; (22):15-18. 
9. Brasil. Ministério da Saúde. Ministério da Educação. Portaria interministerial n. 2101, de 3 de novembro de 2005. Institui o Programa Nacional de Reorientação da Formação Profissional em Saúde - Pró-Saúde para os cursos de graduação em Medicina, Enfermagem e Odontologia. Brasília, DF; 2005. [capturado 12 set. 2014]; Disponível em: http://portal.saude.gov.br/portal/arquivos/ pdf/2101.pdfhttp:// portal.saude.gov.br/portal/arquivos/pdf/2101.pdf

10. Brasil. Ministério da Saúde e Ministério da Educação. Portaria Interministerial n. 3019, de 26 de Novembro de 2007. Dispõe sobre o Programa Nacional de Reorientação da Formação Profissional em Saúde - Pró-Saúde - para os cursos de graduação da área da saúde. Brasília, DF; 2007. [capturado 26 jun 2014]; Disponível em: http: / / bvsms.saude.gov. br/bvs/saudelegis/gm/2007/pri3019_26_11_2007.htmlhttp://bvsms.saude.gov.br/bvs/saudelegis/gm/2007/ pri3019_26_11_2007.html

11. Brasil. Ministério da Saúde e Ministério da Educação. Portaria Interministerial n. 1802, de 26 de agosto de 2008. Institui o Programa de Educação pelo Trabalho para a Saúde - PET - Saúde. [Internet]. Brasília, DF; 2008. [capturado26 jun 2014]. Disponível em: http://bvsms.saude.gov.br/ bvs/saudelegis/gm/2008/pri1802_26_08_2008.html

12. Andrade MRS, Silva CRLD, Silva A, Finco M. Formação em saúde:experiências e pesquisas nos cenários de prática, orientação teórica e pedagógica. Blumenau: Edifurb; 2011.

13. Koltermann PI, Silva ELT. Educação tutorial no ensino presencial: a experiência do PET na UFMS. [capturado 02 jul 2014]. Disponível em: http://portal.mec.gov.br/sesu/arquivos/pdf/PET/pet_texto_i.pdfhttp:/ / portal.mec.gov. $\mathrm{br} / \mathrm{sesu} /$ arquivos/pdf/PET/pet_texto_i.pdfhttp://portal.mec.gov.br/sesu/arquivos/pdf/PET/pet_texto_i.pdf

14. Silveira JLGC, Padilha WWN, Soares EL. A prática de pesquisa como principio educativo. Movimento 2002; 5:111-127.

15. Almeida M. Diretrizes Curriculares Nacionais para os cursos Universitários da Área da Saúde. Londrina, PR: Rede Unida; 2003.

16. Bloom BS. Taxonomia de objetivos educacionais. Porto Alegre: Globo; 1972.

17. Ferraz APCM, Belhot RV. Taxonomia de Bloom: revisão teórica e apresentação das adequações do instrumento para definição de objetivos instrucionais. Gest. Prod. 2010; 17(2):421-431.

18. Machado MFAS, Monteiro EMLM, Queiroz DT, Vieira NFC, Barroso MGT. Integralidade, formação de saúde, educação em saúde e as propostas do SUS - uma revisão conceitual. Ciênc. saúde coletiva 2007; 12(2):335-342.
19. Demo P. Pesquisa e construção de conhecimento. Rio de Janeiro: Tempo Brasileiro; 1996.

20. Brasil. Plano Nacional de Extensão Universitária. Edição Atualizada. Fórum Nacional de Pró-Reitores de Extensão Da Universidades Públicas Brasileiras e SESu/MEC 200/2001. [Internet]. capturado 09 de set 2013]. Disponível em: https:/ / coec.jatai.ufg.br/up/431/o/PNEX.pdf

21. Gomes MPC, Ribeiro VMB, Monteiro DM, Leher EMT, Louzada RCR. O uso de metodologias ativas no ensino de graduação nas ciências sociais e da saúde - avaliação dos estudantes. Ciência\&Edu 2010; 16(1):181-198.

22. Cuff PA. Interprofessional Education for collaboration. Learning how to improve health from Interprofessional models across the continuum of education to Practice. Global Forum on Innovation in Health Professional Education; 2013; Washington: National Academies Press (US); 2013. [capturado 27 jun 2014]. Disponível em: http:/ / www.ncbi. nlm.nih.gov/books/NBK207106/http:/ / www.ncbi.nlm. nih.gov/books/NBK207106/

23. Oliveira Ml, Mendonça MK, Alves Filho HL, Coelho TC, Benetti CN. PET-Saúde: Formar e Fazer como Processo de Aprendizagem em Serviços de Saúde. Rev. Bras. de Edu. Méd 2012; 36(1/2):105-111.

24. Pinto ACM, Oliveira IV, Santos ALS, Silva LES, Izidoro GSL, Mendonça RD, Lopes ACS. Percepção dos alunos de uma universidade pública sobre o Programa de Educação pelo Trabalho para a Saúde. Ciênc. saúde colet. 2013; 18(8): 2201-10.

25. Fonsêca GS, Junqueira SR, Zilbovicius C, Araujo ME. Educação pelo trabalho: reorientando a formação de profissionais da saúde. Interface (Botucatu). 2014;18(50):57185 [capturado11 nov. 2014]; Disponível em: http:/ / issuu. com/revista.interface/docs/interface_v.18_n.50_95030c17 c0cde3/0http:/ /issuu.com/revista.interface/docs/interface_v.18_n.50_95030c17c0cde3/0

26. Santos KT, Ferreira L, Batista RJ, Bitencourt CTF, Araújo RP, Carvalho RB. Percepção discente sobre a influência de estágio extramuro na formação acadêmica odontológica. RevOdontol UNESP 2013; 42(6):420-425.

27. Facchini LA, Nunes BP, Saes MO, Duro SMS, Tomasi E, Siqueira F, Volz PM, Silveira DS, Stofel NS, Wachs LS, Soares UM, Thumé E. Os sentidos da Pesquisa nos processos organizativos da Estratégia Saúde da Família. In: Sousa MF, Franco MS, Mendonça AVM, org. Saúde da Família nos Municípios Brasileiros: os reflexos dos vinte anos no espelho do futuro. Campinas: Saberes; 2014. p. 844-92.

28. Silva AFL, Ribeiro CDM, Silva Júnior SJ. Pensando extensão universitária como campo de formação em saúde: uma 
experiência na Universidade Federal Fluminense, Brasil. Interface (Botucatu). 2013; 17(45):371-84. [capturado 20 nov. 2014]; Disponível em:http:/ / issuu.com/revista.interface/docs/revista46_cd119a2e15afadhttp:/ /issuu.com/ revista.interface/docs/revista46_cd119a2e15afad

29. Moretti-pires RO, Bueno SMV. Freire e formação para o Sistema Único de Saúde: o enfermeiro, o médico e o odontólogo. Acta Paul Enferm 2009; 22(4):439-44.

\section{CONTRIBUIÇÃO DOS AUTORES}

Os autores João Luiz Gurgel Calvet da Silveira e Karla Ferreira Rodrigues participara da concepção do projeto, coleta e análise dos dados e elaboração do artigo científico.

As autoras Mônica Shishido e Patrícia Moraes participaram da coleta e análise de dados e da elaboração do artigo.

\section{CONFLITO DE INTERESSES}

Os autores declaram não haver conflito de interesses, de qualquer natureza, para a participação nas etapas deste projeto.

\section{ENDEREÇO PARA CORRESPONDÊNCIA}

João Luiz Gurgel Calvet da Silveira

Rua São Paulo, 2171 - sala A 302 - Campus 3 - FURB

Bairro Itoupava Seca - Blumenau

CEP 89030-000 - SC

E-mail: gurgeljl@gmail.com 